\title{
Cirugía de cabeza y cuello durante la pandemia de COVID-19 en Colombia: Experiencia de un centro de excelencia
}

\author{
Head and neck surgery during the COVID-19 pandemic in Colombia: \\ Experience from a center of excellence
}

\author{
Martha Castañeda ${ }^{1}$, Jorge Jiménez ${ }^{2} \mathbb{D}$, Carlos Hernando Morales ${ }^{3}$; \\ Juan Guillermo Sánchez $\mathbb{D}^{\mathbb{D}}$, Carlos Andrés García ${ }^{5} \mathbb{D}$, Adriana Isaza $^{4} \mathbb{D}$, David Vargas ${ }^{3} \mathbb{D}$, \\ Álvaro Sanabria ${ }^{5,6} \mathbb{D}$
}

I Odontóloga, especialista en Auditoria Clínica, maestría en Calidad y Sistemas de Gestión, gerente, Centro de Excelencia en Enfermedades de Cabeza y Cuello (CEXCA), Medellín, Colombia

MD, Coordinador de Calidad, Centro de Excelencia en Enfermedades de Cabeza y Cuello (CEXCA), Medellín, Colombia

MD, especialista en Cirugía general, Centro de Excelencia en Enfermedades de Cabeza y Cuello (CEXCA), Medellín, Colombia

MD, especialista en Otorrinolaringología, Centro de Excelencia en Enfermedades de Cabeza y Cuello (CEXCA), Medellín, Colombia

5 MD, Especialista en Cirugía general, subespecialista en Cirugía de Cabeza y Cuello, Centro de Excelencia en Enfermedades de Cabeza y Cuello (CEXCA), Medellín, Colombia

6 Profesor, Departamento de Cirugía, Facultad de Medicina, Universidad de Antioquia, Medellín, Colombia

\section{Resumen}

Introducción. La pandemia por SARS-CoV-2 modificó la prestación de servicios de salud para priorizar el manejo de los casos emergentes. La Asociación Colombiana de Cirugía fue una de las primeras en liderar dichas iniciativas. Este estudio describe el comportamiento de los servicios de salud de un centro especializado en patología de cabeza y cuello en Medellín, Colombia.

Métodos. Este es un análisis retrospectivo de una cohorte prospectiva, en el que se usó la base de datos administrativa. Las variables analizadas fueron el número y tipo de consultas y procedimientos, realizados en el periodo de enerojulio de 2109 y su comparación con el mismo periodo de 2020. Se realizó un análisis descriptivo usando porcentajes, promedio y desviación estándar.

Resultados. Se analizaron 3521 consultas y 866 procedimientos quirúrgicos. Se observó una caída del número de consultas, que alcanzó su punto más bajo en el mes de abril, con un 33,9\% de disminución El porcentaje de consultas de telemedicina aumentó desde marzo de 2020, hasta alcanzar un $98 \%$ en el mes de abril. Se observó una caída del número de procedimientos, con un aumento compensatorio en junio del 62,5 \%. A la fecha, ningún paciente ni integrante del equipo de atención en salud ha presentado infección por CoVID-19.

Fecha de recibido: 11/10/2020 - Fecha de aceptación: 02/11/2020

Correspondencia: Álvaro Sanabria MD, PhD, FACS. Calle 7 \# 39-107, Consultorio 1308. Edificio Medical, Medellín, Antioquia. Teléfono: +574 2669633. Correo electrónico: alvarosanabria@gmail.com

Citar como: Castañeda M, Jiménez J, Morales CH; Sánchez JG, García CA, Isaza A, et al. Cirugía de cabeza y cuello durante la pandemia de COVID-19 en Colombia: Experiencia de un centro de excelencia. Rev Colomb Cir. 2021;36:35-41 https://doi.org/10.30944/20117582.793

Este es un artículo de acceso abierto bajo una Licencia Creative Commons - BY-NC-ND https://creativecommons.org/licenses/by-ncnd/4.0/deed.es 
Discusión. La pandemia de COVID-19 tuvo un efecto importante en el número de consultas y de procedimientos quirúrgicos de cabeza y cuello. La estrategia de telemedicina ayudó a compensar la disminución del acceso a la atención especializada.

Palabras clave: COVID-19; virus del SRAS; pandemias; neoplasias de cabeza y cuello; cirugía general; servicios de salud.

\begin{abstract}
Introduction. The SARS-CoV-2 pandemic modified the provision of health services to prioritize the management of emerging cases. The Colombian Association of Surgery was one of the first to lead these initiatives. This study describes the behavior of the health services of a center specialized in head and neck pathology in Medellín, Colombia.
\end{abstract}

Methods. This is a retrospective analysis of a prospective cohort, in which the administrative database was used. The variables analyzed were the number and type of consultations and procedures, carried out in the period from January to July 2019 and their comparison with the same period in 2020. A descriptive analysis was carried out using percentages, average and standard deviation.

Results. On the database, 3,521 consultations and 866 surgical procedures were analyzed. A drop in the number of consultations was observed, reaching its lowest point in the month of April, with a 33.9\% decrease. The percentage of telemedicine consultations increased since March 2020, reaching 98\% in the month of April. A drop in the number of procedures was observed, with a compensatory increase in June of $62.5 \%$. To date, no patient or member of the health care team has developed a COVID-19 infection.

Discussion. The COVID-19 pandemic had a significant effect on the number of consultations and head and neck surgical procedures. The telemedicine strategy helped to compensate the decrease in access to specialized care.

Keywords: COVID-19; SARS virus; pandemics; head and neck neoplasms; general surgery; health services.

\section{Introducción}

La pandemia por SARS-CoV-2 causó una crisis de salud pública que modificó la prestación de servicios de salud ${ }^{1}$. Las medidas que se tomaron para priorizar el manejo de los casos emergentes implicó el cierre de los servicios ambulatorios y la restricción en la práctica de cirugías electivas ${ }^{2}$. Estas medidas estaban enfocadas en preparar el sistema de salud para la potencial llegada de enfermos graves que ocuparan las camas hospitalarias y de cuidados intensivos.

A pesar del aumento de la incidencia de los casos con SARS-CoV-2, con el paso de los días se ha hecho claro que las demás patologías continúan enfermando la población. Esto es particularmente cierto para la patología oncológica, donde se ha demostrado un retraso en el tratamiento y un efecto pronostico adverso a largo plazo ${ }^{3,4}$. Varias guías publicadas proponen estrategias para el manejo de los pacientes con diagnóstico de cáncer y buscan balancear la atención de los pacientes con COVID-19 y las demás patologías ${ }^{5-7}$.

En Colombia, la Asociación Colombiana de Cirugía fue una de las primeras en liderar dichas iniciativas. Pasadas las primeras semanas de la pandemia, la necesidad de reabrir la atención de los servicios quirúrgicos motivó la aparición de nuevas recomendaciones. A la fecha no se tiene información local sobre experiencias de implementación de una vuelta segura a la atención de los pacientes con patología cervicofacial. Se asume que, mantener los servicios que atienden pacientes con patología potencialmente maligna puede mitigar los efectos de la pandemia por SARS-CoV-2 en el largo plazo.

Este estudio describe el comportamiento de los servicios de salud de un centro especializado 
en patología de cabeza y cuello en Medellín, Colombia y sus resultados a corto plazo.

\section{Métodos}

Este es un análisis retrospectivo de una cohorte prospectiva realizado en el Centro de Excelencia en Enfermedades de Cabeza y Cuello (CEXCA) de Medellín, Colombia, que es un centro especializado que atiende pacientes de patología cervicofacial, benigna y maligna, ambulatoria y hospitalaria, en varias instituciones hospitalarias de la ciudad (Clínica Las Vegas - Grupo Quironsalud, Instituto Colombiano del Dolor INCODOL, Interquirófanos). La base de datos administrativa fue la fuente primaria de los datos.

Las variables analizadas fueron el número y tipo de consultas y procedimientos realizados en el periodo de enero a julio de 2109 , y su comparación con el mismo periodo de 2020 . Se realizó un análisis descriptivo de los datos usando promedio y desviación estándar para las variables continuas y porcentajes y rangos para las variables categóricas.

\section{Resultados}

Se analizaron 3521 consultas y 866 procedimientos quirúrgicos practicados en el Centro de Excelencia en Enfermedades de Cabeza y Cuello (CEXCA).

\section{Consulta}

En el periodo estudiado de 2019 se realizaron 1743 consultas, con un promedio de $249 \pm 26,1$ consultas/mes, mientras que en 2020 se realizaron 1778 consultas, con un promedio de $254 \pm$ 62,9 consultas/mes. La figura 1 muestra el comportamiento por meses, donde se observa una caída progresiva del número de consultas, que alcanzó su punto más bajo en el mes de abril de 2020 , con un 33,9 \% de disminución.

Se realizaron 622 consultas de primera vez en 2019 , con un promedio de consultas de $88,8 \pm$ 7,0 por mes, mientras que en 2020 se realizaron 580 consultas, con un promedio de $82,9 \pm 38,8$ por mes. La mayor disminución ocurrió en el mes de abril de 2020, con un 51,8 \% de disminución (figura 2). De manera similar, se realizaron 1121 consultas de control en 2019 , con un promedio de consultas de $161,5 \pm 26,4$ por mes, mientras que en 2020 se realizaron 1198 consultas, con un promedio de $171,1 \pm 38,3$ por mes. La mayor disminución ocurrió en el mes de abril de 2020, con un 24,8 \% de disminución.

Específicamente derivado de la situación de pandemia, el número de consultas de telemedicina aumentó de manera dramática, hasta alcanzar un $98 \%$ en el mes de abril (figura 3 ).

\section{Procedimientos}

En el periodo estudiado de 2019 se realizaron 418 procedimientos, con un promedio de 59,6 $\pm 12,1$ procedimientos/mes, mientras que en 2020 se realizaron 448 procedimientos, con un promedio de $64,0 \pm 20,4$ procedimientos/mes. La figura 4 muestra el comportamiento por meses, donde se observa una caída del número de procedimientos, que alcanzó su punto más bajo en el mes de febrero de 2020 , con un $26,2 \%$ de disminución. La figura 5 muestra el tipo de procedimientos practicados en el CEXCA y el comportamiento comparativo del número de procedimientos entre los dos periodos.

A la fecha, con 30 días de seguimiento de todos los pacientes operados, ninguno ha presentado infección por COVID-19 y ninguno de los integrantes del equipo de atención han tenido contagio.

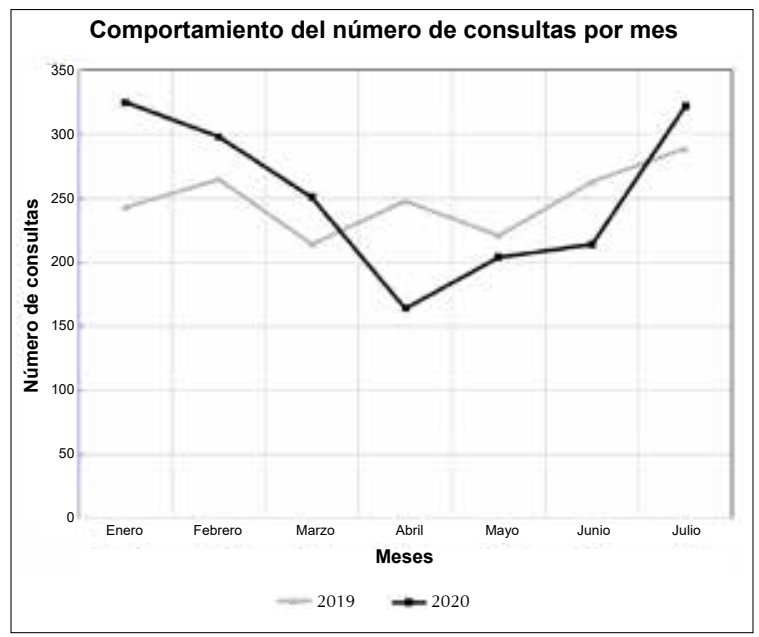

Figura 1. Comportamiento comparativo del número de consultas especializadas en un centro de excelencia en enfermedades de cabeza y cuello 

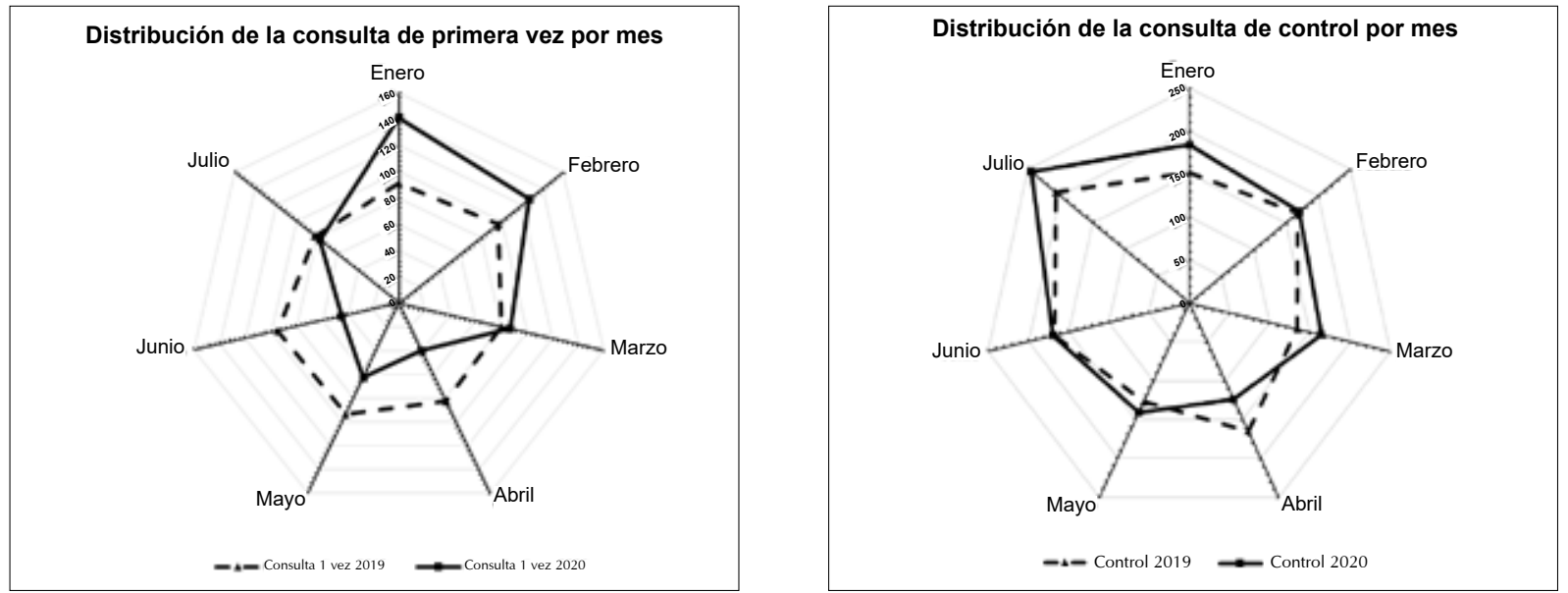

Figura 2. Comportamiento comparativo del número de consultas especializadas, de primera vez y de control, en un centro de excelencia en enfermedades de cabeza y cuello.

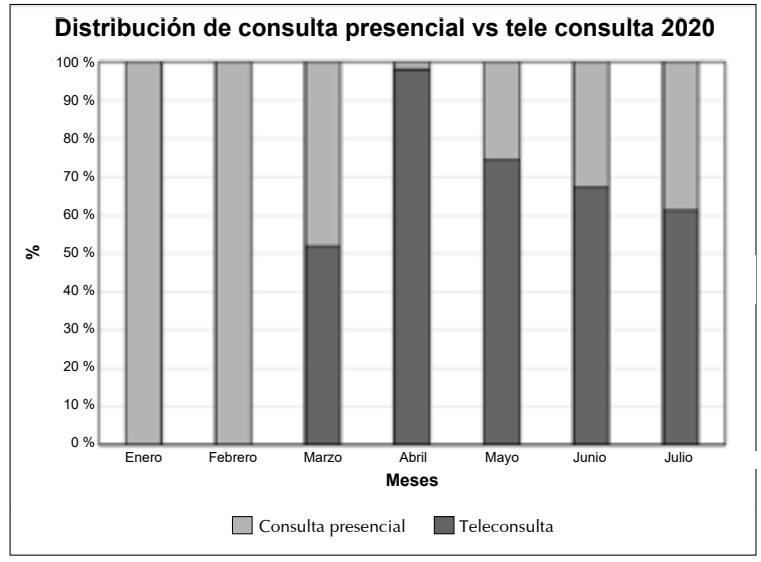

Figura 3. Comportamiento del número de consultas especializadas, presenciales y de telemedicina, en un centro de excelencia en enfermedades de cabeza y cuello durante la pandemia COVID-19.

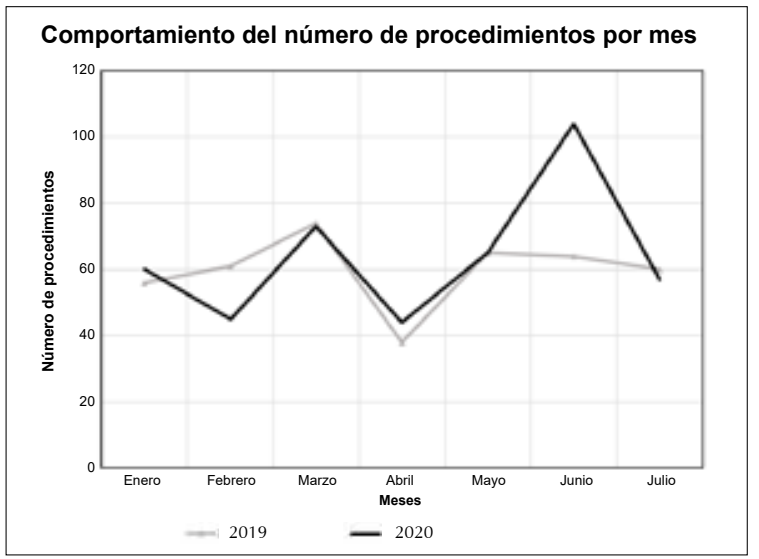

Figura 4. Comportamiento comparativo del número de procedimientos quirúrgicos en un centro de excelencia en enfermedades de cabeza y cuello.

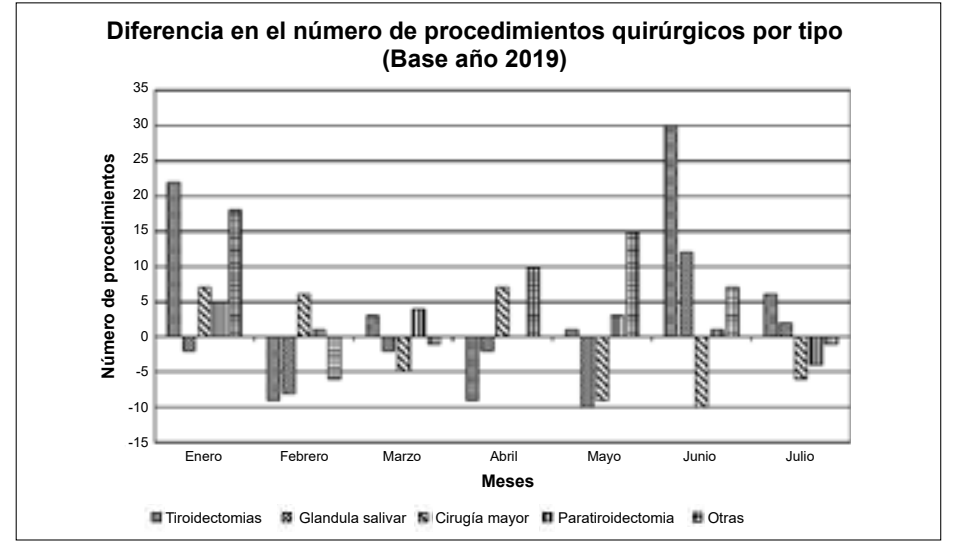

Figura 5. Comportamiento comparativo del tipo de procedimientos quirúrgicos en un centro de excelencia en enfermedades de cabeza y cuello durante la pandemia COVID-19. 


\section{Discusión}

En estos tiempos de incertidumbre, y ante la ausencia de evidencia científica sobre cómo afrontar la pandemia por SARS-CoV-2, los servicios quirúrgicos han tenido que desarrollar estrategias para atender los pacientes con COVID-19 y, al mismo tiempo, no dejar de prestar los servicios quirúrgicos esenciales. Esta situación es particularmente importante para los pacientes con patología potencialmente maligna que requieren tratamiento quirúrgico. A la fecha se sabe que el retraso en el tratamiento de los pacientes oncológicos produce progresión en el estadio de la enfermedad y un empeoramiento en la sobrevida global ${ }^{8}$.

Existen varias experiencias en Europa y Asia que buscaron seleccionar aquellos pacientes oncológicos con alta sospecha de infección, para mantenerlos aislados y alejados de las instituciones que atienden pacientes inmunosuprimidos, $\mathrm{y}$ al mismo tiempo, permitir el acceso a aquellos con necesidades quirúrgicas urgentes que se encontraban sin infección. También existen iniciativas para priorizar a los pacientes con enfermedades potencialmente malignas, definiendo que la cirugía oncológica debe ser la última en ser retrasada; la creación de instituciones no-COVID para la atención de pacientes oncológicos; la creación de planes de contingencia para reiniciar la atención lo más rápido posible, y el establecimiento de planes para reclutar profesionales, que permita atender la demanda acumulada de pacientes que se espera después de pasados los picos de contagio. Estas propuestas pueden servir de ejemplo para los servicios que atienden pacientes con enfermedades cervicofaciales en Colombia ${ }^{8-15}$.

Entre otras medidas, nuestro centro estableció protocolos de tamización preclínica, con cuestionarios específicos al momento de asignar la cita y al momento de confirmar la cita, y tamización clínica, al momento de la atención del paciente, con medición de temperatura y signos vitales. Se restringió el acompañamiento de familiares solo para casos especiales, como pacientes ancianos o con discapacidad evidente, se adecuó la infraestructura para garantizar el aislamiento social y se limitó la participación de los estudiantes durante la consulta y los procedimientos quirúrgicos.
Simultáneamente, se elaboró una estrategia en redes sociales reforzando las medidas de autocuidado y mensajes telefónicos por los encuestadores, además de la estrategia de teleconsulta para aquellos pacientes que se encontraban en seguimiento y con riesgo bajo de recurrencia, que permitió protegerlos de una exposición innecesaria, mientras se mantuvo la atención presencial para pacientes de primera vez y en controles posquirúrgicos inmediatos. Para ciertos tumores, como los tiroideos de bajo riesgo, la estrategia de vigilancia activa fue ampliamente aplicada en cuestión de semanas. Los casos de pacientes con tumores donde una espera razonable fuera posible, por ejemplo, tumores de glándulas salivares, fueron reagendados, esperando la evolución de la pandemia y la disponibilidad de camas hospitalarias y camas de UCI.

En paralelo, también se desarrolló una estrategia de protección del personal de salud, con educación en los procedimientos de protección y aislamiento social, y el uso rutinario de elementos de protección personal (EPP). En este caso, el uso de la teleconsulta también ayudó a disminuir el riesgo de contagio para el personal administrativo $\mathrm{y}$ asistencial.

En un principio, fueron limitados completamente los procedimientos diagnósticos (como nasofibrolaringoscopia o biopsia percutánea, entre otros), pero con el tiempo, el mayor conocimiento y el uso adecuado de los EPP, estos fueron reasumidos, disminuyendo el tiempo de espera para la definición de un diagnóstico y el inicio del tratamiento adecuado.

Con esto se logró mantener el flujo de atención en los niveles mas altos posibles. Los resultados de esta cohorte muestran que la aplicación juiciosa de las recomendaciones y el respeto estricto por ellas permite mantener en límites razonables la atención de los pacientes con enfermedades potencialmente malignas de cabeza y cuello.

Aunque es evidente una caída en el número de actividades, la implementación de la teleconsulta compensó parcialmente las limitaciones de la atención presencial. La consulta presencial sufrió una caída dramática en los meses con mayor restricción de la movilidad y mayor limitación por la 
cuarentena, pero la consulta de control se mantuvo en niveles aceptables, como consecuencia de la implementación de la teleconsulta. Existe información sobre la alta satisfacción de los pacientes con el uso de esta herramienta, aunque no dejan de existir dificultades relacionadas con la realización del examen físico y cierta ansiedad por la falta de contacto físico entre paciente y médico ${ }^{16}$.

El número de procedimientos quirúrgicos también sufrió una caída, aunque la variación dependió del país, el tipo de práctica y el momento de la pandemia ${ }^{17,18}$. La estrategia desarrollada permitió que esta diminución no fuera tan dramática, lo que contrasta con reportes de supresión de casi el $100 \%$ de la practica quirúrgica en hasta el $17 \%$ de los servicios ${ }^{18}$. No obstante, el tipo de procedimientos realizados han tenido variaciones. En ciertos periodos la cirugía mayor fue radicalmente restringida, dada la falta de disponibilidad de camas de cuidados intensivos, mientras que los procedimientos diagnósticos fueron privilegiados al no necesitar de mayor recurso hospitalario.

En conclusión, nuestra experiencia demuestra que, realizando adaptaciones clínicas y administrativas basadas en la evidencia, fue posible enfrentar la restricción de los servicios quirúrgicos para garantizar una atención oportuna a los pacientes con enfermedades de cabeza y cuello.

\section{Cumplimiento de normas éticas}

Consentimiento informado. La fuente primaria de los datos fue una base de datos administrativa. Debido a que no se utilizan datos específicos de pacientes, no se requirió de consentimiento informado.

Conflicto de interés. Ninguno declarado por los autores.

Financiación. El estudio fue financiado con recursos propios de los autores.

Contribución de los autores. Diseño; adquisición, análisis e interpretación de datos; redacción y revisión crítica: Martha Castañeda, Álvaro Sanabria, Jorge Jiménez.

Adquisición, interpretación de datos y revisión crítica: Carlos Morales, Juan Guillermo Sánchez, Carlos Andrés García, Adriana Isaza, David Vargas.

Todos los autores leyeron y aprobaron el manuscrito final.

\section{Referencias}

1. Hartley DM, Perencevich EN. Public health interventions for COVID-19: Emerging evidence and implications for an evolving public health crisis. JAMA. 2020;323:19089. https://doi.org/10.1001/jama.2020.5910

2. Barrios A, Prieto R, Torregrosa L, Álvarez C, Hernández J, González L, et al. Volver a empezar: cirugía electiva durante la pandemia del SARS-CoV2. Recomendaciones desde la Asociación Colombiana de Cirugía. Rev Colomb Cir. 2020;35:302-21.

https://doi.org/10.30944/20117582.656

3. Chen-See S. Disruption of cancer care in Canada during COVID-19. Lancet Oncol. 2020;21:e374. https://doi.org/10.1016/S1470-2045(20)30397-1

4. Maringe C, Spicer J, Morris M, Purushotham A, Nolte E, Sullivan R, et al. The impact of the COVID-19 pandemic on cancer deaths due to delays in diagnosis in England, UK: a national, population-based, modelling study. Lancet Oncol. 2020;21:1023-34.

https://doi.org/10.1016/S1470-2045(20)30388-0

5. Burki TK. Cancer guidelines during the COVID-19 pandemic. Lancet Oncol. 2020;21:629-30. https://doi.org/10.1016/S1470-2045(20)30217-5

6. Garg PK, Kaul P, Choudhary D, Turaga KK, Singh MP, Tiwari AR, et al. Discordance of COVID-19 guidelines for patients with cancer: A systematic review. J Surg Oncol. 2020;122:579-93. https://doi.org/10.1002/jso.26110

7. Tartarone A, Lerose R. COVID-19 and cancer care: what do international guidelines say? Med Oncol. 2020;37:80.

https://doi.org/10.1007/s12032-020-01406-5

8. Tzeng CD, Teshome M, Katz MHG, Weinberg JS, Lai SY, Antonoff MB, et al. Cancer surgery scheduling during and after the COVID-19 first wave: The MD Anderson Cancer Center experience. Ann Surg. 2020;272:e106-e111. https://doi.org/10.1097/SLA.0000000000004092

9. Resteghini C, Maggioni P, Di Martino V, Licitra L. Head and Neck Cancer Medical Oncology Unit, Fondazione IRCCS Istituto Nazionale dei Tumori Milan, Italy. Response of the Istituto Nazionale Tumori of Milan Head \& Neck Cancer Unit to the COVID-19 outbreak. Cancers Head Neck. 2020;5:7. https://doi.org/10.1186/s41199-020-00054-6

10. de Almeida JR, Noel CW, Forner D, Zhang H, Nichols $\mathrm{AC}$, Cohen MA, et al. Development and validation of a surgical prioritization and ranking tool and navigation aid for head and neck cancer (SPARTAN-HN) in a scarce resource setting: Response to the COVID-19 pandemic. Cancer. 2020 Aug 11. https://doi.org/10.1002/cncr.33114

11. Anwar SL, Harahap WA, Aryandono T. Perspectives on how to navigate cancer surgery in the breast, head and neck, skin, and soft tissue tumor in limited-resour- 
ce countries during COVID-19 pandemic. Int J Surg. 2020;79:206-12.

https://doi.org/10.1016/j.ijsu.2020. 05.072

12. Fakhry N, Schultz P, Moriniere S, Breuskind I, Bozece A, Vergez $\mathrm{S}$, et al. French consensus on management of head and neck cancer surgery during COVID-19 pandemic. Eur Ann Otorhinolaryngol Head Neck Dis. 2020;137:159-60.

https://doi.org/10.1016/j.anorl.2020.04.008

13. Givi B, Schiff BA, Chinn SB, Clayburgh D, Iyer G, Jalisi S, et al. Safety recommendations for evaluation and surgery of the head and neck during the COVID-19 pandemic. JAMA Otolaryngol Head Neck Surg. 2020;146:579-84. https://doi.org/10.1001/jamaoto.2020.0780

14. Mehanna H, Hardman JC, Shenson JA, Abou-Foul AK, Topf MC, AlFalasi M, et al. Recommendations for head and neck surgical oncology practice in a setting of acute severe resource constraint during the COVID-19 pandemic: an international consensus. Lancet Oncol. 2020;21:e350-e359.

https://doi.org/10.1016/S1470-2045(20)30334-X

15. Finley C, Prashad A, Camuso N, Daly C, Aprikian A, Ball $\mathrm{CG}$, et al. Guidance for management of cancer surgery during the COVID-19 pandemic. Can J Surg. 2020;63:S2S4. https://doi.org/10.1503/cjs.005620

16. Triantafillou V, Layfield E, Prasad A, Daly C, Aprikian A, Ball CG, et al. Patient perceptions of head and neck ambulatory telemedicine visits: A qualitative study. Otolaryngol Head Neck Surg. 2020:194599820943523. https://doi.org/10.1177/0194599820943523

17. Zaid W, Schlieve T. The early effects of coronavirus disease-2019 on head and neck oncology and microvascular reconstruction practice: A national survey of oral and maxillofacial surgeons enrolled in the head and neck special interest group. J Oral Maxillofac Surg. 2020;78:1859-68.

https://doi.org/10.1016/j.joms.2020.07.012

18. Kowalski LP, Imamura $\mathrm{R}$, de Castro-Junior G, Nader-Marta G, Freitas-Chaves AL, Luongo-Matos L, Ferreira-Bento R. Effect of the COVID-19 pandemic on the activity of physicians working in the areas of head and neck surgery and otorhinolaryngology. Int Arch Otorhinolaryngol. 2020;24:e258-e266.

https://doi.org/10.1055/s-0040-1712169 